\title{
Untersuchungen über den Einfluß der Salze auf die Wachstumsrichtung der Wurzeln, zunächst an der Erbsenwurzel.
}

Von H. C. Schellenberg, Zürich.

(Mit zwei Textfiguren).

Die Orientierungsbewegungen der Pflanzen werden durch verschiedene äußere Lebensbedingungen hervorgerufen. Licht, Schwerkraft, stoffliche Verschiedenheiten des Mediums, mechanische Einflüsse vermögen am Pflanzenkörper Wachstums- und Bewegungserscheinungen auszulösen, die für das Pflanzenleben zweckdienlich erscheinen. Wir bezeichnen diese Einflüsse als Reize und Aufgabe der Physiologie ist es, den kausalen Zusammenhang zwischen dem Reiz und der Orientierungsbewegung in dem komplizierten Lebensgetriebe nachzuweisen. Wenn auch in den letzten Jahrzehnten die Reizphysiologie von den Botanikern intensiv gepflegt wurde und sehr schöne Resultate zutage förderte, so stehen wir leider über den ursächlichen Zusammenhang von Reiz und Bewegung überall noch auf dem Ǵebiet der Hypothesen, von denen wir sagen können, daß sie mehr oder weniger den Tatsachen entsprechen werden. Jeder Beitrag, der neue Tatsachen liefert, ist ein wertvoller Baustein für den weiteren Ausbau der Reizphysiologie; aber auch jede Hypothese, die sich auf die Tatsachen stützt, ist beachtenswert, indem sie zu neuer Forschung Anregung gibt. So soll auch diese Arbeit in erster Linie Bausteine für die Erkenntnis des Reizvorganges, den die Salze ausüben, liefern und wenn ich mir erlaube, eine Hypothese über den Vorgang auszusprechen, so geschieht das nur in der Absicht, zu weiteren Untersuchungen anzuregen.

Ausgangspunkt für meine Untersuchungen waren die Erscheinungen des Galvanotropismus, der an der Phanerogamenwurzel zuerst von Elfving entdeckt wurde. Wenn eine wachsende Wurzel im Wasser zwischen zwei Elektroden gebracht wurde, so fand Elfving, daß die Wurzel in dem weiteren Wachstum nach dem positiven Pole abgelenkt wurde. Bei einigen Pflanzen, so bei Brassica, beobachtete aber Elfving Krümmung nach dem negativen Pole. 
Müller-Hettlingen hat die Frage weiter untersucht und konnte die Elfvingschen Angaben bestätigen. Zugleich zeigte er aber, daß wenn der Versuch mit Brassica in anderer Weise angeordnet wird, dann auch diese Wurzel sich dem positiven Pole zuwendet. Ebenso gelang es Müller-Hettlingen bei einer Anzahl von Pflanzen, die sich nach dem positiven Pole wendeten, auch die Krümmung nach dem negativen Pole zu erzielen.

Brunchorst ${ }^{1,2}$ ) hat dann besonders die Einwirkung verschiedener Stromstärken auf den Galvanotropismus genauer untersucht. Als Resultat findet er, daß die, Krümmung der Wurzeln abhängig von der Stromstärke ist, indem bei schwächeren Strömen die Krümmung nach der negativen Elektrode, bei stärkeren Strömen nach der positiven Elektrode stattfindet. Die Grenze zwischen negativen und positiven Krümmungen liegt bei verschiedenen Pflanzen bei verschiedener Stromstärke. Brunchorst untersucht dann weiter die Frage nach der Funktion der Wurzelspitze in der galvanotropischen Ablenkung der Wurzel. Er findet, daß die Spitze allein die empfindliche Region bei der negativen Krümmung ist. Die positive Krümmung werde dagegen durch Schädigungen, die auf der ganzen Länge der Wurzel stattfinden, herbeigeführt. Sie stellt nach Brunchorsts Ansicht eine ganz andere Kategorie von Erscheinungen dar als die negative Krümmung und muß als eine durch die chemische Wirkung des Stromes verursachte Krankheits- und Absterbeerscheinung aufgefaßt werden.

In einer späteren Arbeit wendet sich Brunchorst gegen eine Auffassung von Rischavi, der im Stoffaustausch die Ursachen der galvanotropischen Krümmungen sucht und präzisiert seinen Standpunkt genauer.

Diese Versuche stellen ziemlich alles dar was auf dem Gebiete des Galvanotropismus der Wurzelspitzen geleistet worden ist. Die neueren Anschauungen über Dissoziation und die Leitung des elektrischen Stromes konnten noch nicht berücksichtigt werden. Zweifelsohne hätte die Dissoziationstheorie Anlaß zu anderer Fragestellung geben müssen.

Wenn nun der Galvanotropismus nicht weiter verfolgt wurde, so zeigen doch die Untersuchungen über den Chemotropismus mancherlei Angaben, die mit der elektrolytischen Dissoziation in Zusammenhang zu bringen sind. Die ersten Beobachtungen nach dieser Richtung stammen von Pfeffer. Er fand bei seinen Untersuchungen über den Chemotropismus der Farnspermatozoiden, daß Äpfelsäure in intensiver Weise den Reiz ausübt und ebenso die Salze der Äpfelsäure, während die Ester diese Wirkung nicht ausüben. Diese Tatsache weist wohl unzweideutig darauf hin, daß der Chemotropismus in irgend einer Be- 
ziehung zur Dissoziation dieser Körper und damit zu den elektrischen Eigenschaften der Lösungen steht.

In einer weiteren Arbeit hat Buller, ein Schüler Pfeffers, auf dessen Anregung diesen Gegenstand weiter verfolgt. Er fand, daß die Objekte, welche einen ausgeprägten Chemotropismus für bestimmte Substanzen aufweisen, daneben in schwächerem Maße sich durch andere Salze verschiedener Stoffe auch chemotropisch reizen lassen. Dieser Befund weist wiederum darauf hin, daß die chemotropische Reizung in einer Eigenschaft zu suchen ist, die allgemein den Salzen zukommt und weniger von der Natur der Substanz abhängig ist. Auch in den Arbeiten von W. Rothert findet sich manche Angabe, die auf solche Verhältnisse hinweist.

Diese Beobachtungen veranlaßten mich, Reizversuche mit Salzen wieder aufzunehmen, nachdem durch $\mathrm{Ne}$ e co m be und Rhodes und Lilienfeld in einer vorläufigen Mitteilung darauf aufmerksam gemacht wurde, daß die Phanerogamenwurzel allgemein durch Salze gereizt werden kann. Seit dem Beginn meiner Arbeit ist die ausführliche Arbeit von Lilienfeld erschienen; ferner die ausgezeichnete Arbeit von Shibàta über den Chemotropismus der Isoïtesspermatozoiden, weiterhin eine Arbeit von R. Sammet über Chemotropismen an Wurzeln, Sprossen und Pilzfäden und von B. Lidfors über Chemotropismus der Marchantiaspermatozoiden. Sie alle haben neues Material gebracht und gezeigt, daß die Wirkungen der Salze nicht von den osmotischen Leistungen abhängig sind, sondern auf den Erscheinungen der Dissoziation beruhen und als Leistungen der Ionen aufgefasst werden können.

Da Brunchorst den starken Strömen eine wachstumshemmende Wirkung zuschreibt, wollte ich diese Erscheinungen außer Betracht lassen und nur die Effekte der schwachen Ströme studieren, denn diese können ja allein bei der Erklärung chemotroper Erschênungen in Frage kommen.

Ich habe denn' auch mit dem gleichen Erfolg wie dieser Autor seine Versuche wiederholt.

Erbsen- und Bohnenkeimlinge, deren Wurzeln völlig gerade gewachsen waren und eine Länge von $5-8 \mathrm{~cm}$ erreicht hatten, wurden in einem kleinen Bassin, das mit Brunnenwasser gefüllt wurde, in lotrechter Richtung aufgehängt. Auf zwei gegenüberliegenden Seiten wurden Elektroden mit der Flüssigkeit in Verbindung gebracht und von einer Akkumulatorenbatterie der Strom durchgeleitet. Die Stromstärke der Batterie war 6 Volt, der durch das System hindurchtretende Strom 0,0001 Ampère. Bereits nach 12 Stunden waren die sämtlichen 
Wurzelspitzen nach der Anode abgelenkt, die sich in den folgenden 12 Stunden noch weiter verstärkt hatte. Parallelversuche in Brunnenwasser, aber ohne Durchleitung eines Stromes zeigten, daß diese regelmäßige Ablenkung der Wurzeln unterblieb. Mit Erbsen und Bohnen war der gleiche Erfolg des Versuches zu verzeichnen.

In welcher Weise ist nun das Ergebnis dieses Versuches weiter $\mathrm{zu}$ verwerten?

Nach den Untersuchungen von van't Hoff wissen wir, daß chemisch reines Wasser zu den Nichtleitern für Elektrizität gehört. Das Wasser wird nur zum Leiter, wenn in demselben Salze oder Säuren gelöst sind. In unserem Falle müssen wir, weil Strom durch das Brunnenwasser durchgegangen ist, dieses Wasser als eine stark verdünnte Lösung von verschiedenen Salzen ansehen. Die Dissoziationstheorie von Arrheniusvan't Hoff sagt nun weiter, daßi $n$ wässerigen Lösungen die Salze in ihre Ionen getrennt sind, und zwar wächst die Trennung in die Ionen mit dem Grade der Verdünnung. Wenn ein elektrischer Strom durch eine Lösung hindurch geleitet wird, so sind diese Ionen die Überträger der Elektrizität. Die Leitfähigkeit einer Lösung für den elektrischen Strom kann als die Summe der Leitfähigkeit der Ionen angesehen werden. Da aber diese Ionen selbst elektrisch geladene Teilchen sind, so wandern sie in den Lösungen, sobald ein Strom hindurchgeht, und zwar die positiv geladenen Teilchen an den negativen Pol und umgekehrt die negativ geladenen Teilchen an den positiven Pol.

Für die weitere Untersuchung gestaltet sich darum die Frage so: wie verhalten sich diese Wurzeln, wenn man an Stelle der unbestimmten Lösung des Brunnenwassers Lösungen von Salzen verwendet?

Da mit Brunnenwasser, das eine sehr stark verdünnte Lösung Jarstellt, positive Erfolge erzielt wurden, so verwendete ich für die weiteren Versuche ebenfalls stark verdünnte Salzlösungen, Konzentrationen, wie sie sich im Bodenwasser wohl finden dürften und von denen anzunehmen ist, daß sie keine schädigende Wirkung auf die Wurzeln ausüben. Giftige Stoffe waren von vornherein auszuschließen, indem solche Wirkungen nur die anderen Ergebnisse getrübt hätten.

Als Versuchspflanze wählte ich die Erbse (Viktoria-Erbsen) und führte mit dieser Versuchspflanze die weitaus meisten Versuche aus. Die Samen wurden in feuchten Sägespänen zur Keimung gebracht und zu den Versuchen verwendet. nachdem die Wurzel 3-4 cm Länge erreicht hatte. Die Versuchsanordnung ist aus der nachstehenden Figur ersichtlich (Fig. 1). Die Versuchspflanzen wurden in lotrechter Richtung aufgehängt, so daß die Wurzel einige Zentimeter in die Lösung 
tauchte, die Kotyledonen aber oberhalb der Flüssigkeit sich befanden. In der Lösung selbst durfte durch den elektrischen Strom keine Zersetzung eintreten. Ich wählte deshalb folgende Elektrode. In einem kleinen Gefäß wurde der Draht in eine Lösung des gleichen Salzes gebracht, wenn auch in etwas stärkerer Konzentration. Mittelst Fließpapierbrücken, die mit der zu untersuchenden Lösung getränkt wurden, stellte ich die Verbindung her; dabei stellte ich das Niveau aller drei Gefäße auf die gleiche Höhe ein, damit ein Überfließen der Flüssigkeiten nicht eintrat. Die Zersetzung der Salze trat nun in den Nebengefäßen ein, während im Hauptgefäße diese während der Versuchsdauer völlig unterblieb und diese Lösung ihre Zusammensetzung nicht änderte. In dem Fließpapierbogen trat eine Bewegung von Salzteilchen so langsam ein, daß der Übertritt der Salze nach 12 Stunden nicht erfolgt war. (Mit farbigen Salzlösungen ist diese Tatsache leicht festzustellen.)

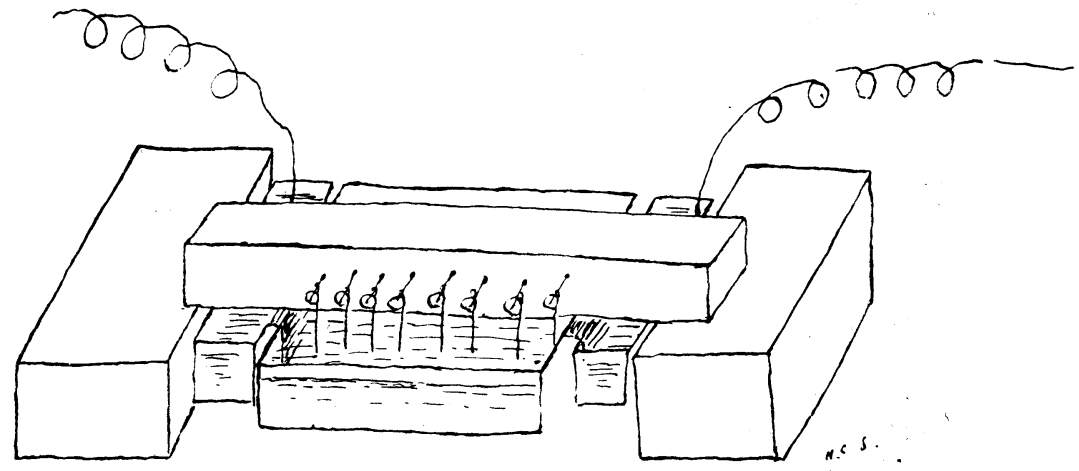

Fig. 1.

Die Versuchsdauer von 12 Stunden war völlig genügend, um recht deutliche Ausschläge in der Wachstumsrichtung der Wurzel zu erhalten. Der ganze Versuch wurde dunkel gehalten, um den Lichteinfluß abzuhalten. Nachdem meine Vorversuche dargetan hatten, daß bereits geringe Elektrizitätsmengen genügten, um die Ablenkung der Wurzeln herbeizuführen, verwendete ich für die meisten Versuche Akkumulatorenbatterien von 2 Volt, 4 Volt und 6 Volt. Der hindurchgetretene Strom war wegen der sehr großen Widerstände sehr gering und war in den meisten Fällen 0,0001-0,000001 Ampère ${ }^{1}$ ). Die Lösungen wurden in gleichmolekularer Konzentration gewählt unter der

1) Ein Stundenampère scheidet $1,184 \mathrm{~g} \mathrm{CuO}$ ab. Meine Stromstärken sind deshalb etwa 100 mal schwächer, als die von Brunchorst in seinen Versuchen angewendeten schwachen Ströme. Der schwächste von Brunchorst gemessene Strom ist 0,14 $\mathrm{mg} \mathrm{Cu}$ pro Stunde, was 0,00011 Amp. entspricht. 
Annahme, daß bei dieser Verdünnung die Dissoziation des Salzes vollständig ist, was, auch der Wirklichkeit nach den Tabellen über elektrische Leitfähigkeit von Kohlrausch nahe kommen soll. Ich wählte als Vergleich eine Konzentration von Kochsalz, bei welcher auf $100 \mathrm{ccm}$ Wasser 0,02 g Kochsalz kommen. Die Vorversuche hatten ergeben, daß bei dieser Konzentration die Wurzeln regelmäßig gegen die Anode, den Ort, wo sich das positive Metallteilchen abscheidet, hinwendet.

Das Ergebnis dieser Versuchsreihe findet sich in folgender Tabelle zusammengestellt:

\begin{tabular}{|c|c|c|c|c|c|c|}
\hline \multirow[t]{2}{*}{ Salz } & \multirow[t]{2}{*}{$\begin{array}{l}\text { Molekular- } \\
\text { gewicht }\end{array}$} & \multicolumn{2}{|c|}{$\begin{array}{l}\text { Konzentration. } \\
\text { Auf } 100 \mathrm{~g} \text { Wasser sind } \\
\text { Salz enthalten }\end{array}$} & \multicolumn{3}{|c|}{$\begin{array}{c}\text { Erfolg. } \\
\text { Die Wurzeln wachsen zur }\end{array}$} \\
\hline & & Gramm & Gramm-Molekul. & Anode & Kathode & indiff. \\
\hline $\mathrm{KCl}$ & 74,4 & 0,025 & 0,00034 & 10 & - & - \\
\hline KJ & 173,8 & 0,056 & 0,00033 & 8 & - & 2 \\
\hline $\mathrm{KNO}_{2}$ & 101 & 0,034 & 0,00033 & 8 & - & 2 \\
\hline $\mathrm{K}_{2} \mathrm{SO}_{4}^{3}$ & 173,8 & 0,039 & 0,00022 & 9 & - & 1 \\
\hline $\mathrm{K}_{2}^{2} \mathrm{HPO}_{4}$ & 152,2 & 0,035 & 0,00022 & 8 & 1 & 1 \\
\hline $\mathrm{NaCl}^{2}$ & 58,5 & 0,020 & 0,00034 & 8 & 1 & 1 \\
\hline $\mathrm{Na}, \mathrm{SO}_{+}$ & 174,2 & 0,039 & 0,00022 & 10 & - & - \\
\hline $\mathrm{NaNO}_{3}$ & 85 & 0,029 & 0,00034 & 9 & - & 1 \\
\hline $\mathrm{Na}_{3} \mathrm{PO}_{4}$ & 164 & 0,028 & 0,00017 & 10 & - & - \\
\hline $\mathrm{NH}_{4} \mathrm{Cl}^{+}$ & 53,3 & 0,019 & 0,00034 & 2 & 6 & 2 \\
\hline $\mathrm{NH}_{4}^{4} \mathrm{NO}_{3}$ & 80 & 0.027 & 0,00033 & 6 & 2 & 2 \\
\hline$\left(\mathrm{NH}_{4}\right)_{2} \mathrm{SO}_{4}^{3}$ & 132 & 0,029 & $0,000 \cdot 2$ & 7 & 1 & 2 \\
\hline$\left(\mathrm{NH}_{3}\right)_{2} \mathrm{HPO}_{4}$ & 132 & 0,029 & 0,00022 & 8 & 1 & 1 \\
\hline $\mathrm{MgCl}_{2}$ & 95 & 0,021 & 0,00022 & 9 & - & 1 \\
\hline $\mathrm{MgSO}_{4}$ & 120 & 0,041 & 0,00033 & 8 & - & 2 \\
\hline $\mathrm{Mg}\left(\mathrm{NO}_{3}^{+}\right)_{2}$ & 148 & 0,032 & 0,00022 & 9 & - & - \\
\hline $\mathrm{SrCl}_{2}$ & 152,6 & 0,035 & 0,00022 & 9 & - & 1 \\
\hline $\mathrm{Sr}\left(\mathrm{NO}_{3}\right)_{2}$ & 211,5 & 0,048 & 0,00022 & 9 & - & 1 \\
\hline $\mathrm{Cg}(\mathrm{NO})^{3}$ & 164 & 0,037 & 0,00022 & 10 & - & - \\
\hline
\end{tabular}

Wenn man die Ammoniumsalze einstweilen außer der Betrachtung läßt, so zeigt sich, daß die Wurzel in allen andern Fällen auf die Seite der Metallabscheidung abgelenkt wird. Daraus könnte man, wie MüllerHettlingen es aus seinen Versuchen getan hat, schließen, daß die Richtung des elektrischen Stromes maßgebend für die Ablenkung der Wachstumsrichtung der Wurzel gewesen ist. Es würde dann die Wurzel in der Stromrichtung abgelenkt. Allein die Tatsache, daß bei den Ammoniumsalzen eine Ablenkung in der entgegengesetzten Richtung eintritt, beweist, daß hier noch andere Verhältnisse im Spiel sein müssen. Aus den Beobachtungen über chemotropische Bewegungen ergibt sich ferner, daß nicht allein Bewegungen in der Richtung des Konzentrationsgefälles, sondern auch solche entgegen dieser Richtung vorkommen. Die chemotropische Bewegung ist unter Umständen in der Richtung umkehrbar. In unseren Versuchen müßte also die Wachstumsrichtung der Wurzel unter gewissen Bedingungen umkehrbar sein. 
Die Versuche wurden nun bei gleicher Konzentration der Lösungen so angestellt, daß die Richtung des elektrischen Stromes gewechselt wurde. Alle diese Versuche zeigten, daß mit dem Wechsel der Stromrichtung auch die Wachstumsrichtung der Wurzel wechselte und zwar in dem Sinne, daß die Wurzel immer nach der Anode sich wendete. Auch bei den Ammoniumsalzen wurde mit dem Wechsel der Stromrichtung auch die Wachstumsrichtung der Wurzel geändert, so daß die Ablenkung der Wachstumsrichtung immer in gleicher Richtung zum Strom erfolgte. Also auch diese Versuche hätten nur ergeben, daß die Wachstumsrichtung der Wurzel abhängig von der Stromrichtung ist.

Da aber die chemotropischen Versuche ergeben hatten, daß die Konzentration der Lösungen maßgebend für die Richtung der chemotropischen Bewegung war, so war es selbstverständlich geboten, Versuche mit Lösungen verschiedener Konzentrationen auszuführen.

Schon die erste Versuchsserie zeigte, dah bei gleicher Stromrichtung und Stromintensität die Wachstumsrichtung der Wurzel abhängig von der Konzentration der Lösung ist. Erbsen in Chlorkaliumlösungen.

$\begin{array}{rc}\begin{array}{r}\text { Konzentration } \\ \text { in } 100 \mathrm{H}_{2} \mathrm{O}\end{array} & \begin{array}{c}\text { Zahl der } \\ \text { Versuchspflanzen }\end{array} \\ 0,025 \mathrm{~g} & 10 \\ 0,035 \mathrm{~g} & 10 \\ 0,074 \mathrm{~g} & 10 \\ 0,123 \mathrm{~g} & 10 \\ & \\ 0,185 \mathrm{~g} & 10 \\ 0,243 \mathrm{~g} & 10 \\ 0,352 \mathrm{~g} & 10 \\ 1.00 \mathrm{~g} & 10\end{array}$

Mit der Steigerung der Konzentration fand eine Umwendung der Wachstumsrichtung statt und zwar zwischen 0,2 und $0,4 \% \mathrm{KCl}$. Es ist leicht einzusehen, daß individuelle Verschiedenheiten zwischen den einzelnen Exemplaren es nicht möglich machten, eine scharfe Grenze zu bestimmen. Für das gleiche Exemplar dürfte die Umstimmung innerhalb eines geringeren Intervalles erfolgen.

Nachdem einmal an einem Salz gezeigt worden war, daß bei gleicher Stromrichtung eine Änderung in der Wachstumsrichtung der Wurzel herbeigeführt werden konnte, war es unmöglich, den elektrischen Strom verantwortlich für die Veränderung zu machen, sondern ich mußte 
annehmen, daß es Beziehungen zwischen der Konzentration der Außenlösung und den Verhältnissen der lebenden Zellen der Wurzeln sind, in welchen dieser Reiz einwirkt, die von maßgebender Bedeutung für diese Umstimmung sind. Es war mir deswegen wichtig, durch Versuche diese Konzentration der Umstimmung für eine Reihe von Salzen an demselben Objekt $\mathrm{zu}$ bestimmen. In folgenden Tabellen lasse ich die Resultate meiner Versuche folgen. Sie sind alle unter gleichen Bedingungen angestellt worden bei Zimmertemperatur 6 Volt. Batterie und Stromdurchgang 0,0001-0,0000001 Ampère. Ich will noch besonders hervorheben, daß in keinem einzigen Versuche die durchgeflossene Strommenge mehr als 0,0002 Ampère betrug, sondern meistens schwankte die Strommenge von 0,00001-0,000001. Wenn also hier eine Umstimmung in der Wurzel zustande kam, so kann sie nicht als ein Erfolg der größeren durchgeflossenen Strommenge aufgefaßt werden, weil mit der Konzentrationserhöhung die Leitfähigkeit der Lösung besser wurde. Wie man sieht, sind meine Strommengen viel schwächer, als die von Brunchorst verwendeten Größen, von denen er selbst sagt, daß sie keine Schädigung der Wurzeln herbeiführen. Die Stromstärke für die Umstimmung, d. h. für das Auftreten der positiven Krümmung lag bei den Versuchen Brunchorsts zwischen 4 und $16 \mathrm{mg} \mathrm{Cu}$ pro Stunde $=0,005-0,013$ Ampère, also bei Stromstärken, die im Mittel 1000 mal größer sind als die in meinen Versuchen im Durchschnitt zur Verwendung gelangten Ströme.

I. KJ. Molekulargewicht 166.

\begin{tabular}{cl} 
Konzentration & \multicolumn{1}{c}{ Gramm } \\
$\%$ Molekule \\
0,056 & 0,00034 \\
0,132 & $(1,000795$ \\
0,189 & 0,00114 \\
0,315 & 0,00196
\end{tabular}

\begin{tabular}{ccc}
\multicolumn{3}{c}{ Ablenkung der Wurzeln nach } \\
Anode & Kathode & indifferent \\
8 & - & 2 \\
5 & 4 & 1 \\
1 & 5 & 2 \\
1 & 9 & 2
\end{tabular}

II. $\mathrm{KNO}_{3}$. Molekulargewicht 101 .

$\begin{array}{lllll}0,034 & 0,00033 & 8 & - & 2 \\ 0,10 & 0,00099 & 8 & - & -1 \\ 0,143 & 0,00141 & 6 & 1 & 2 \\ 0,333 & 0,00329 & 5 & 3 & 4 \\ 0,500 & 0,00495 & 2 & 4 & 3 \\ 0,600 & 0,00594 & 3 & 4 & \end{array}$

III. $\mathrm{K}_{2} \mathrm{SO}_{4}$. Molekulargewicht 173,8 .

$\begin{array}{lllll}0,02 & 0,00011 & 4 & - & 1 \\ 0,039 & 0,00022 & 9 & - & 1 \\ 0,12 & 0,00068 & 4 & 2 & 2 \\ 0,34 & 0,0019 & 2 & 6 & 2 \\ 0,68 & 0,0039 & 2 & 4 & 1 \\ 092, & 0,0052 & 2 & 4 & 2 \\ 1,36 & 0,0072 & 3 & 4 & 1\end{array}$


IV. $\mathrm{K}_{2} \mathrm{HPO}_{4}$. Molekulargewicht 152,2.

Konzentration

$\%$

0,035

0,081

0,60

0,68

0,78
Gramm

Moleküle

0,00022

0,00053

0,00393

0,00446

0.00513
Ablenkung der Wurzeln nach

$\begin{array}{ccc}\text { Anode } & \text { Kathode } & \text { indifferent } \\ 8 & 1 & 1 \\ 9 & - & 1 \\ 8 & - & 0 \\ 8 & - & 2 \\ 1 & 6 & 2\end{array}$

V. NaCl. Molekulargewicht 58,5.

$\begin{array}{llccc}0,020 & 0,00034 & 8 & 1 & \cdot \\ 0,023 & 0,00039 & 9 & - & 1 \\ 0,039 & 0,00066 & 6 & 1 & 1 \\ 0,047 & 0,00080 & 8 & 2 & 3 \\ 0,055 & 0,00094 & 7 & 2 & 1 \\ 0,097 & 0,00165 & 2 & 6 & 2 \\ 0,105 & 0,00179 & 1 & 6 & 3 \\ 0,163 & 0,002-8 & - & 6 & 2\end{array}$

VI. $\mathrm{NaNO}_{3}$. Molekulargewicht 85 .

$\begin{array}{lllll}0,029 & 0,00034 & y & - & 1 \\ 0,080 & 0,00094 & 5 & - & 1 \\ 0,121 & 0,00142 & 9 & 1 & - \\ 0,210 & 0,00247 & 6 & 2 & 2 \\ 0,425 & 0,00500 & 2 & 6 & 1 \\ 0,460 & 0,00541 & 1 & 6 & 2\end{array}$

VII. $\mathrm{Na}_{2} \mathrm{SO}_{4}$. Molekulargewicht 174,2 .

0,039

0,052

0,090

0,174

0,205

0,00022

0,000298

0,000516

0,00100

0,00117

10
8
8
1
1

$\frac{-}{\overline{9}}$

2
2
1
2

VIII. $\mathrm{NH}_{4} \mathrm{Cl}$. Molekulargewicht 53,3.

0,007
0,013
0,019
$0,(0) 5$
0,030
0,035
0,132
0,215
0,530

0,00013

0,00024

0,00034

0,00047

0,00056

0,00065

0,00248

0,00403

0,00994

5
4
6
1
1
1
2
3
-

1
3
2
8
8
6
5
4
4

$$
\begin{aligned}
& 2 \\
& 2 \\
& 2 \\
& 1 \\
& 2 \\
& 2 \\
& 1 \\
& 1 \\
& 6
\end{aligned}
$$

IX. $\mathrm{NH}_{4} \mathrm{NO}_{3}$. Molekulargewicht $8 \dot{0}$.

0,012
0,027
0,030
0,035
0,050
0,072
0,113
0,189
0,330
0,530

0,00015

0,00031

0,00037

0,00043

$0,(; 0062$

0,00090

0,00141

$0,00: 36$

0,00412

0,00662

$\begin{array}{rr}8 & - \\ 6 & 2 \\ - & 7 \\ 2 & 6 \\ - & 10 \\ - & 6 \\ 2 & 7 \\ - & 6 \\ 2 & 5 \\ - & 4\end{array}$

\begin{tabular}{l}
-2 \\
2 \\
2 \\
\hline 2 \\
0 \\
3 \\
1 \\
6
\end{tabular}


X. $\left(\mathrm{NH}_{4}\right)_{2} \mathrm{SO}_{4}$. Molekulargewicht 132 .

$\begin{array}{cc}\begin{array}{c}\text { Konzentration } \\ \%\end{array} & \begin{array}{c}\text { Gramm } \\ \text { Moleküle }\end{array} \\ 0,020 & 0,00015 \\ 0,029 & 0,00022 \\ 0,034 & 0,00025 \\ 0,053 & 0,00040 \\ 0,074 & 0,00056 \\ 0,330 & 0,00250 \\ 0,330 & 0,00250 \\ 0,528 & 0,00400 \\ 0,655 & 0,00496\end{array}$

Ablenkung der Wurzeln nach Anode Kathode indifferent

XI. $\left(\mathrm{NH}_{4}\right)_{2} \mathrm{HPO}_{4}$. Molekulargewicht 132 .

$\begin{array}{lllll}0,029 & 0,00022 & 8 & 1 & 1 \\ 0,030 & 0,00022 & 6 & 1 & 2 \\ 0,052 & 0,00039 & 7 & - & 2 \\ 0,080 & 0,00060 & 1 & 6 & 2 \\ 0,120 & 0,00909 & 1 & 7 & 2\end{array}$

XII. $\mathrm{MgCl}_{2}$. Molekulargewicht 95 .

$\begin{array}{llrrr}0,021 & 0,(0022 & 9 & - & 1 \\ 0,040 & 0,00042 & 10 & - & - \\ 0,174 & 0,00138 & 10 & 1 & 1 \\ 0,850 & 0,00894 & 1 & 6 & 2 \\ 1,42 & 0,0149 & 1 & 7 & 3\end{array}$

XIII. $\mathrm{MgSO}_{4}$. Molekulargewicht 120 .

$\begin{array}{llrrr}0,041 & 0,00033 & 8 & - & 2 \\ 0,112 & 0,00093 & 12 & - & 1 \\ 0,180 & 0,00110 & 5 & 1 & 1 \\ 0,450 & 0,00375 & 8 & 3 & 1 \\ 0,600 & 0,00500 & 6 & 3 & 1 \\ 0,900 & 0,00750 & 3 & 4 & 3 \\ 1,80 & 0,01500 & 1 & 8 & \end{array}$

XIV. $\operatorname{Mg}\left(\mathrm{NO}_{3}\right)_{2}$. Molekulargewicht 148 .

$\begin{array}{lllll}0,032 & 0,00022 & 9 & - & 1 \\ 0,315 & 0,00213 & 8 & 2 & 1 \\ 0,737 & 0,00498 & 4 & 5 & 1 \\ 1,105 & 0,00745 & 2 & 6 & 2 \\ 2,210 & 0,01493 & -- & 6 & 2\end{array}$

$\mathrm{XV} . \mathrm{CaCl}_{2}$. Molekulargewicht 111.
0,236
0,00212
9
1

Bei höheren Konzentrationen zeigen die Wurzeln kein Wachstum mehr.

XVI. $\mathrm{Ca}\left(\mathrm{NO}_{3}\right)_{2}$. Molekulargewicht 164.

$\begin{array}{llrrr}0,037 & 0,00022 & 10 & - & - \\ 0,245 & 0,00149 & 10 & - & - \\ 0,359 & 0,002189 & 6 & 1 & 4 \\ 1,220 & 0,00743 & 3 & 4 & 2\end{array}$

XVII. $\mathrm{SrCl}_{2}$. Molekulargewicht 152,6.

$\begin{array}{lllll}0,035 & 0,00022 & 9 & - & 1 \\ 0,044 & 0,000282 & 9 & - & 1 \\ 0,147 & 0,000963 & 4 & 4 & 2 \\ 0,211 & 0,00138 & 1 & 7 & 2 \\ 0,336 & 0,002208 & 1 & 6 & 3\end{array}$


XVIII. $\quad \operatorname{Sr}\left(\mathrm{NO}_{3}\right)_{2}$. Molekulargewicht 211,5.

\begin{tabular}{clccc} 
Konzentration & Gramm & \multicolumn{3}{c}{ Ablenkung der Wurzeln nach } \\
$\%$ & Moleküle & Anode & Kathode & indifferent \\
0,048 & 0,00022 & 9 & 0 & 1 \\
0,208 & 0,000964 & 5 & 2 & 2 \\
0,313 & 0,00148 & 10 & - & - \\
0,413 & 0,00195 & 3 & 6 & 1
\end{tabular}

Aus diesen Zahlen geht zunächst hervor, daß die sämtlichen untersuchten Salze die Erscheinung der Umstimmung aufweisen mit der Änderung der Konzentration der Lösung. Bei einigen Stoffen trat mit der Erhöhung der Konzentration eine Herabsetzung des Wachstums ein. Andere Salze, wie Baryumsalze, wirken direkt giftig, indem die Wurzeln darin rasch auch bei niedrigen Konzentrationen absterben. Solche Stoffe können für die Untersuchung nicht in Betracht fallen, sondern ich beschränkte mich auf diejenigen Wirkungen, bei welchen noch ein gutes Wachstum zu sehen war, denn sonst könnte der Einwand erhoben werden, daß es sich lediglich um Wachstumsstörungen durch den Strom handeln würde.

Wir sehen, daß die Konzentrationsgrenze für die Umstimmung nicht bei allen Stoffen gleich ist, sondern erhebliche Abweichungen aufweist. Auch wenn man nur die Anzahl der Moleküle betrachtet, die durch die Gramm-Moleküle einen Ausdruck finden, so zeigen sich die Unterschiede nur noch deutlicher. In stark verdünnten Lösungen sind die Moleküle in ihre elektrisch geladenen Teile gespalten. Die Ionenzahl wäre deshalb der einzige Vergleichsmaßstab, der zulässig wäre. Ich habe deshalb in nachfolgender Tabelle nach diesen Verhältnissen die Konzentrationen der Umstimmung zusammengestellt unter der Annahme, daß die Moleküle völlig in die Ionen gespalten seien.

Die Umstimmung findet statt:

$\begin{array}{lccl}\text { Ionenzahl } & \begin{array}{c}\text { Gramm } \\ \text { Molekül in } \\ 100 \mathrm{ccm}\end{array} & \begin{array}{c}\text { Gramm } \\ \text { Moleküle, }\end{array} \\ \mathrm{KJ} & 2 & 0,0008 & 0,0004 \\ \mathrm{KCl} & 2 & 0,003-0,005 & 0,002 \\ \mathrm{KNO} & 2 & 0,003-0,005 & 0,002 \\ \mathrm{~K}_{2} \mathrm{SO}_{4} & 3 & 0,0007-0,002 & 0,0004 \\ \mathrm{~K}_{2} \mathrm{HPO}_{4} & 3 & 0,004-0,005 & 0,0015 \\ \mathrm{NaCl}_{4} & 2 & 0,0009-0,0016 & 0,0012 \\ \mathrm{NaNO}_{3} & 2 & 0,002-0,005 & 0,002 \\ \mathrm{Na}_{2} \mathrm{SO}_{4} & 3 & 0,0005-0,001 & 0,00025 \\ \mathrm{NH}_{4} \mathrm{Cl} & 2 & 0,0005 & 0,00025 \\ \mathrm{NH}_{4} \mathrm{NO}_{3} & 2 & 0,00035 & 0,0002 \\ \left(\mathrm{NH}_{4}\right)_{2} \mathrm{SO}_{4} & 3 & 0,00056 & 0,00018 \\ \left(\mathrm{NH}_{4}\right)_{2} \mathrm{HPO}_{4} & 3 & 0,0004-0,0006 & 0,0002 \\ \mathrm{MgCl}_{2} & 3 & 0,001-0,008 & 0,0015 \\ \mathrm{Mg}\left(\mathrm{NO}_{3}\right)_{2} & 3 & 0,005 & 0,0015 \\ \mathrm{MgSO} & 2 & 0,005-0,007 & 0,003 \\ \left.\mathrm{Ca}_{4} \mathrm{NO}_{3}\right)_{2} & 3 & 0,007 & 0,002 \\ \mathrm{SrCl}_{2} & 3 & 0,0009-0,001 & 0,0003 \\ \mathrm{Sr}\left(\mathrm{NO}_{3}\right)_{2} & 3 & 0,0014-0,0019 & 0,0017 \\ & & & \end{array}$


Aus der Tabelle ergibt sich nun, daß die Umstimmung keineswegs nur proportional der Ionenzahl geht. Die Abweichungen sind größer, als allfällige Versuchsfehler das Resultat beeinflußt haben könnten, wenn ich unumwunden auch zugebe, daß in den Experimenten kleinere Abweichungen durch Stehenlassen der Lösungen, ungenügendes Abtrocknen der'Keimlinge, kleine Verdunstung während der Versuchsdauer etc. wohl in geringem Maße zustande gekommen sein können.

Es ist also nicht die Zahl der Ionen allein für das Zustandekommen der Umstimmung maßgebend, sondern der Stoff, aus dem die Ionen bestehen. Damit ist wohl genügend die Ansicht von Brunchorst widerlegt, daß die positive Krümmung allein durch die Ausscheidung des Stoffes an der Kathode zustande kommen soll.

Immerhin, so unregelmäßig, wie es auf den ersten Blick scheint, sind diese Zellen doch nicht. Bei jedem Metall steigt die Konzentration der Ionen für die Umstimmung $\mathrm{I}<\mathrm{SO}_{4}<\mathrm{PO}_{4}<\mathrm{Cl}<\mathrm{NO}_{3}$. Es sind nur zwei geringe Ausnahmen beim $\mathrm{NH}_{4}$, wo die Zahl für das Chlorid etwas größer ist als für das Nitrat und beim Magnesium, wo $\mathrm{SO}_{4}$ die größte Zahl aufweist.

Ebenso zeigt sich, daß bei gleichen Säureionen die Zahlen regelmäßig zunehmen in der Reihenfolge $\mathrm{NH}_{4}<\mathrm{Na}<\mathrm{K}$, wobei sich keine Ausnahme findet. Müßte man $\mathrm{Mg}$ und $\mathrm{Sr}$ in die Reihe einfügen, so würde sie lauten $\mathrm{NH}_{4}<\mathrm{Sr}<\mathrm{Mg}<\mathrm{Na}<\mathrm{K}$.

In einer kleinen Tabelle zusammengestellt, bekommt man folgende Anordnung bei steigenden Werten für die Umstimmung von oben nach unten und links nach rechts.

\begin{tabular}{|llll}
\multicolumn{1}{c}{} & $\mathrm{NH}_{4}$ & $\mathrm{Na}$ & $\mathrm{K}$ \\
\hline $\mathrm{I}$ & & & $\mathrm{KI}$ \\
$\mathrm{SO}_{4}$ & $\left(\mathrm{NH}_{4}\right)_{2} \mathrm{SO}_{4}$ & $\mathrm{Na}_{2} \mathrm{SO}_{4}$ & $\mathrm{~K}_{2} \mathrm{SO}_{4}$ \\
$\mathrm{PO}_{4}$ & $\left(\mathrm{NH}_{4}\right)_{2} \mathrm{HPO}_{4}$ & $\left(\mathrm{Na}_{2}\right) \mathrm{HPO}_{4}$ & $\left(\mathrm{~K}_{2}\right) \mathrm{HPO}_{4}$ \\
$\mathrm{Cl}_{4}$ & $\mathrm{NH}_{4} \mathrm{Cl}$ & $\mathrm{NaCl}_{4}$ & $\mathrm{KCl}$ \\
$\mathrm{NO}_{3}$ & $\mathrm{NH}_{4} \mathrm{NO}_{3}$ & $\mathrm{NaNO}_{3}$ & $\mathrm{KNO}_{3}$
\end{tabular}

Was sagt uns nun diese Tabelle? Sicherlich doch nichts anderes, als daß für die Umstimmung der Erbsenwurzel die Ionen in der Weise maßgebend sind, als daß wir darin die Summe der spezifischen Kationenwirkung plus Summe der spezifischen Anionenwirkungen vor uns haben. Weder, das Kation für sich allein, noch das Anion für sich allein bewirkt in einem Salze die Reizung und die Umstimmung: beide Teile wirken zusammen. 
Aus solchen Zusammenstellungen ist leicht auch die Wirkung eines Salzes, das nicht näher geprüpft worden ist, vorauszusagen, sofern ein anderes Salz bereits in seiner Wirkung bestimmt ist.

Ähnlich gesetzmäßiges Verhalten der verschiedenen Salze ist zum Teil auch aus den Versuchen von Shibata (pag. 590) an Isoëtesspermatozoiden herauszulesen. Für Isoëtes steigen die Konzentrationen für die Umstimmung in der Reihe von $\mathrm{PO}_{4}<\mathrm{SO}_{4}<\mathrm{NO}_{3}<\mathrm{Cl}$ bei allen Metallen. Dagegen glaubt Shibata (pag. 590), daß für die verschiedenen Metalle die gleichen Konzentrationen gelten. Immerhin sind seine Zahlen wie diejenigen von Lilienfeld und Sammet für solche Zwecke nicht wohl zu gebrauchen, indem die angewendete Methode nicht besonders geeignet ist, die Konzentration der Umstimmung genau festzustellen.

Neben den Salzen.zeigen auch die freien Säuren und Basen die Umstimmung bei Änderung der Konzentration. Es ist merkwürdig, daß die Ammoniumsalze bei den niedrigsten Konzentrationen die. Umstimmung bewirken durchschnittlich bei $10 \mathrm{mal}$ geringer Konzentration als die Kalisalze. Für das biologische Verhalten der Wurzeln würde man schließen, daß die Ammoniumsalze ein spezifisches Reizmittel der Erbsenwurzel sind.

\section{Der Einfluß der Stromstärke.}

Während durch meine Versuche dargetan wurde, daß auch eine Krümmung der Wurzeln nach dern positiven Pole bei ganz schwachen Stromstärken erzielt werden kann, wenn die Konzentration der Lösung nur erhöht wird, sagt Brunchorst, daß die Wendung nach dem positiven Pole immer durch stärkere Ströme bedingt sei. Es war deswegen wichtig, wenigstens an einem Salze den Einfluß der Stromstärke näher zu studieren.

Ich wählte dazu $\mathrm{NH}_{4} \mathrm{Cl}$ und $\mathrm{KCl}$.

Der Strom betrug 24 Volt; durchgeflossene Strommenge 0,012 Amp.

$\mathrm{NH}_{4} \mathrm{Cl} \quad 0,01 \%$

8 Wurzeln

$\mathrm{NH}_{4} \mathrm{Cl} 0,01 \%$

Stromstärke 6 Volt; durchgeflossene Strommenge 0,0001 Amp.

8 Wurzeln

$\mathrm{NH}_{4} \mathrm{Cl} \quad 0,01 \%$

Stromstärke 2 Volt; durchgeflossene Strommenge 0,00002-0,00004 Amp.

8 Wurzeln nach 24 Stdn. sind 4 Wurzeln abgestorben. keine abgestorbenen Wurzeln.
$=14,1 \mathrm{mg} \mathrm{Cu}$ pro Stde.

7 stark nach der Kathode, 1 indifferent: Wachstum gering,

6 stark nach dem negativen Pol, 2 indifferent, Wachstum gut,

7 stark nach der Anode gewendet, 1 indifferent, Wachstum gut, keine abgestorbenen Wurzeln. 
Nimmt man stärkere Konzentration $0,1 \% \mathrm{NH}_{4} \mathrm{Cl}$, so bemerkt man, daß 0,01 Amp., 0,0001 und 0,00002 Amp. die Wurzeln nach der Kathode wachsen und keine Umkehrung der Wachstumsrichtung zeigen. Bei 0,01 Amp. sind zahlreiche Wurzeln abgestorben und es zeigen die übrigen Wurzeln ein geringes Wachstum.

Auch die Versuche mit $\mathrm{KCl}$ geben ein gleiches Resultat.

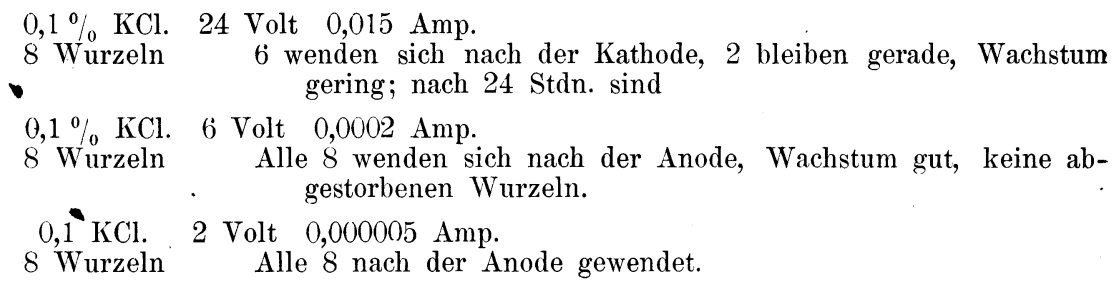

Nimmt man zu den Versuchen eine stärkere Konzentration der Lösung $0,5 \%$, so daß auch bei schwächeren Strömen eine Wendung zur Kathode eintritt, so tritt keine Veränderung in der Wachstumsricutuug auf bei 0,005 Amp. und 0,00001 Amp.; die Wachstumsintensität ist bei dem stärkeren Strom eine sehr geringe. Ebenso zeigen sich bald abgestorbene Wurzeln, während bei dem schwachen Strome die Wurzel in ganz normaler Weise weiter wächst und keine nennenswerte Störung der Wachstumsintensität zeigt.

Diese Versuche zeigen, daß bei Stromstärken unter 0,0001 Ampère - die Wendung zur Kathode nicht durch eine Schädigung des Stromes herbeigeführt wird. Diese Krümmung bei starken Strömen ist deshalb verschieden von der positiven Krümmung, die bei schwachen Strömen, aber größeren Konzentrationen eintreten.

Bei der Frage nach der Änderung der Konzentration der Umstimmung der Wurzeln bei verschiedenen Stromintensitäten können nur die schwachen Ströme mit ihren Effekten in Betracht fallen unter 0,001 Amp. Ich habe zwar diese Frage speziell nicht genauer geprüft, doch scheint es mir nach dem vorliegenden Materiale, daß diese Konzentration der Umstimmung sich nicht wesentlich verschiebt zwischen 0,0001 und 0,00001 Ampère, wenigstens haben mir einige Parallelversuche, wo bei gleichen Batterien der Widerstand erhöht wurde, annähernd gleiche Resultate gegeben für die Konzentration der Umstimmung.

Dagegen scheint die Intensität des Stromes - innerhalb gewisser Grenzen - die Intensität der Wachstumsänderung zu beeinflussen. Während bei Einschaltung grosser Widerstände, wo die durchgeflossene Strommenge unter 0,000001 Amp. betrug, die Reaktion der Wurzel 
nur langsam sich einstellte, sowohl bei höheren oder niedrigeren Salzkonzentrationen, trat bei 0,00001-0,0001 Amp. die Reaktion rascher ein.

\section{Der Ort der Reizperzeption.}

Für den Geotropismus ist zuerst von Darwin gezeigt worden, daß die Wurzelspitze in der Hauptsache den Reiz empfindet. Dieser Befund ist durch zahlreiche spätere Untersuchungen bestätigt worden. Man hat nun auch für den Lichtreiz die gleiche Verteilung der Empfindlichkeit gefunden. Brunchorst hat die Verteilung der Empfindlichkeit auf den galvanotropischen Reiz genauer studiert. Er findet, daß die Krümmung zur Anode ähnlich wie die Schwerkraft in der Wurzelspitze empfunden wird; dagegen wird die positive Krümmung in der ganzen Länge der Wurzel wahrgenommen.

Meine Untersuchung hatte in erster Linie festzustellen, ob diese beiden verschiedenen Krümmungen auch in ungleicher Weise von der Wurzel empfunden werden. Ich machte deswegen folgenden Versuch:

$\mathrm{KCl} 0,1 \%=0,0009 \mathrm{GM}$.

24 Volt $0,01 \mathrm{Amp} .=11,8 \mathrm{mg} \mathrm{Cu}$ pro Stde.

Wurzeln intakt

12 Wurzeln n. d. Kathode gewendet.

" $1 \mathrm{~mm}$ von der Spitze algeschnitten 6 , alle n. d. Kathode gewendet.

, $2, \quad, \quad, \quad$,

, $3, \quad, \quad, \quad, \quad$,

" $4, \quad, \quad$,

Dieser Versuch stimmt mit den Angaben von Brunchorst überein. Die Entfernung eines mehr oder weniger großen Stückes von der Wurzelspitze hat diese Krümmung nicht gehindert. Daraus folgere ich wie Brunchorst. daß der Reiz in der wachstumsfähigen Zone ebenfalls empfunden wird, und zwar ebensogut wie in der Spitze. Ich halte die Ursache dieser Erscheinung für eine Schädigung, die längs der ganzen Wurzel eintritt. Es zeigen solche Wurzeln ein sehr geringes Wachstum, ebenso treten bald unter den am meisten gebogenen Exemplaren abgestorbene Individuen auf. Die Versuchsanordnung von Brunchorst (II, p. 195) beweist. ferner, daß diese Schädigung auf der Seite des + Poles intensiver auftritt. Die Ursache der Schädigung sieht Brunchorst im Kation, das allein wirke, weil es zur Ausscheidung gelangt. Gegenwärtig dürfte man diese Erscheinungen aus der elektrischen Polarisation am besten erklären.

Verschieden von dieser bezeichnet Brunchorst I die Krümmung nach dem negativen Pole, die durch schwache Ströme hervorgerufen wird. „Diese hat viel mehr Ähnlichkeit mit den Richtungsbewegungen 
wie andere. Es findet dabei kein Absterben der Wurzeln statt; sie wachsen ruhig weiter, ob auch nicht so gut wie nicht im Strome befindliche, und machen, wenn der Strom nicht mehr auf sie einwirkt, normale geotropische Krümmungen. Es liegt deshalb bis jetzt nichts vor, was eine andere Auffassung wie die, sie als eine den Richtungsbewegungen ganz analoge Krümmung zu betrachten, notwendig macht."

Aus seinen Dekapitierungsversuchen der Wurzelspitzen schließt Brunchorst weiter, „daß bei der negativen Krümmung die Spitze allein die empfindliche Region ist, von welcher aus der Reiz auf die obere Region übertragen wird". Ebenso schließt Müller-Hettlingen aus seinen Versuchen, daß vorzugsweise die Wurzelspitze den Reiz empfindet, wenn die Wurzel nach dem negativen $\mathrm{Pol}$ sich wendet.

Daraus geht nun aber noch nicht hervor, ob die Wendung der Spitze ;um positiven Pole, wie sie durch Erhöhung der Salzkonzentration auch - bei Anwendung sehr schwacher Ströme eintritt, auch in der ganzen Länge der Wurzelspitze oder nur besonders in der Spitze empfunden wird.

Ich habe deshalb die Dekapitierungsversuche wiederholt, und zwar mit dem gleichen Salz bei niedriger Konzentration, die eine Wendung der Wurzel zur Anode und bei höherer Konzentration der Lösung zur Kathode herbeiführte.

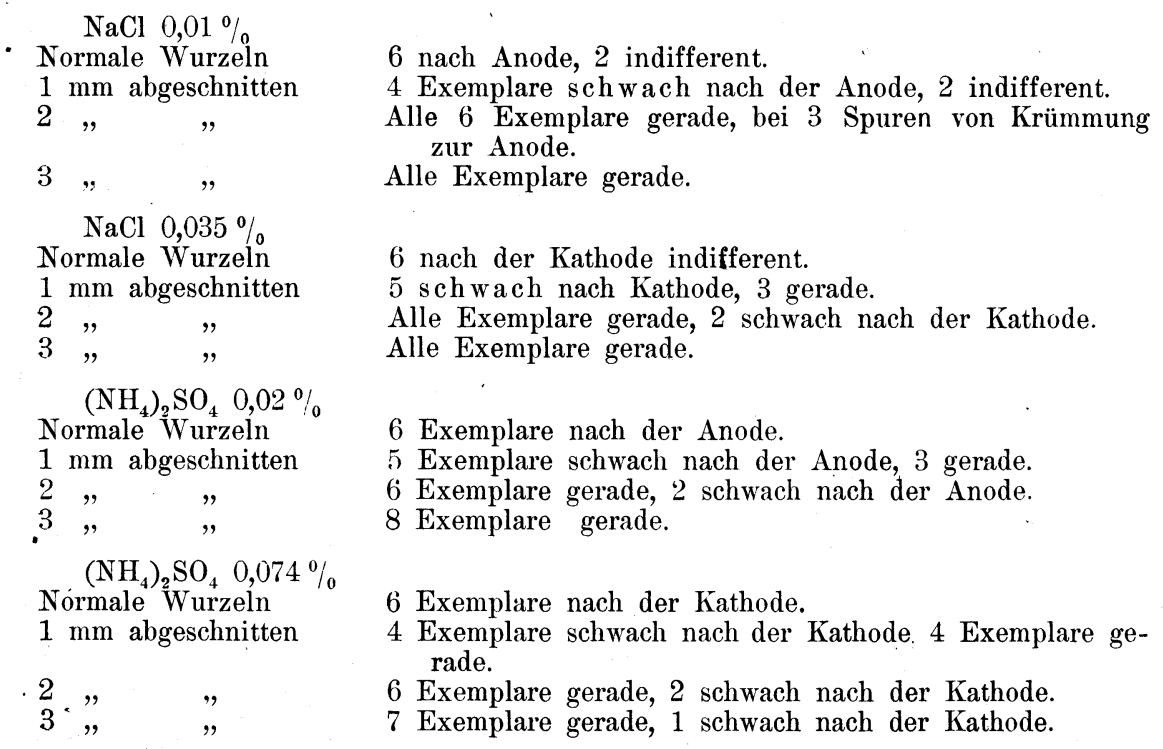

Diese beiden Versuchsserien für Natrium und Ammoniumsalze bestätigen das Ergebnis Brunchorsts, daß vor allem die Wurzelspitze das empfindliche Organ für die Krümmung nach dem - Pol ist. AnderFlora 1906. 
seits zeigen sie aber, daß entgegen der Annahme Brunchorsts die positive Krümmung bei schwachem Strom durch die Wurzelspitze empfunden wird, genau so wie die negative Krümmung. Es existiert zwischen der Verteilung der Empfindlichkeit für die negative Krümmung und die positive Krümmung keine nennenswerte Differenz bei beiden geprüften Salzen. Daraus folgere ich, daß bei Verwendung von schwachen Strömen eine Schädigung längs der ganzen Wurzel nicht in erheblichem Maße eintritt und daß unter diesen Bedingungen positive wie negative Krümmung an den gleichen Orten empfunden wird.

Weiter muß aber aus diesen Versuchen geschlossen werden, wie das Brunchorst getan hat, daß diese Krümmungen bei schwachen Strömen verschieden sind von der positiven Krümmung, die bei starken Strömen auftreten und nicht miteinander in die gleiche Kategorie von Erscheinungen gestellt werden. Das betrifft auch die positive Krümmung, die bei schwachen Strömen infolge der Erhöhung der Konzentration der Lösung eintritt.

\section{Zur Theorie chemotropischer Erscheinungen.}

Von sämtlichen Forschern wird die Ablenkung der Phanerogamenwurzel in ihrer Wachstumsrichtung durch Konzentrationsgefälle von Salzlösungen als eine chemotropische Erscheinung angesprochen. Als verschieden davon hat man die Ablenkung der Wurzeln durch den galvanischen Strom angesehen und ihn als Galvanotropismus bezeichnet. Beide Erscheinungen zeigen so viel äußerliche Ähnlichkeiten, daß man wohl die Frage diskutieren darf, ob der Chemotropismus der Salze und der Galvanotropismus nicht auf die gleichen Ursachen zurückzuführén sind. Besonders die neueren Anschauungen über die Dissoziation und die Leitfähigkeit der Salzlösungen weisen den Weg, wie man sich den Zusammenhang dieser Erscheinungen etwa zu denken hat und geben auch Anhaltspunkte zur Prüfung der Erscheinungen.

Brunchorst, der sich mit dem Galvanotropismus der Wurzeln befaßt hat, erklärt die eine Ablenkung der Wachstumsrichtung der Wurzeln aus der elektrischen Polarisation. Sie tritt nach seiner Auffassung bei den entgegengesetzten Flanken ungleich ein; dadurch werde eine ungleiche Hemmung des Wachstums auf den entgegengesetzten Seiten der Wurzel herbeigeführt und somit komme die Abbiegung der Wurzel zustande. Dieser Auffassung kann ich mich anschließen, nur soweit es die Wirkungen starker elektrischer Ströme betrifft. Immerhin dürfte auch aus der Elektrizitätslehre eine neue Basis zum näheren Verständnis der Erscheinung gegeben werden. 
Die folgenden Untersuchungen beziehen sich nur auf die Effekte schwacher Ströme, von denen Brunchorst bereits bemerkt, daß sie den Richtungsbewegungen, die durch Schwerkraft und Licht hervorgerufen werden, analoge Erscheinungen sind.

Der Effekt, den der elektrische Strom in einer Lösuhg erzeugt, ist die Ionenwanderung. Weder das Wasser noch der gelöste Elektrolyt, z. B. die Schwefelsäure leitet im absolut reinen Zustand den elektrischen Strom; die Leitfähigkeit erscheint erst mit der Lösung des einen Körpers in dem andern. Mit der Lösung tritt gleichzeitig die Spaltung des Salzes in die beiden elektrisch geladenen Teile, die Ionen auf. Diese Trennung in die Ionen vergrößert sich mit dem Grade der Verdünnung und ist bei starker Verdünnung vollständig. Die Leitfähigkeit einer Lösung für den elektrischen Strom ist abhängig von der Zahl der Ionen und ist gleich Leitfähigkeit der Kationen plus Leitfähigkeit der Anionen. Wichtig für unsere Betrachtung ist dann ferner das Ergebnis, daß der Strom nicht die Ionisierung eines Salzes in der Lösung erhöht oder erniedrigt, sofern die Ionen nicht abgeschieden werden, sondern nur die bereits bestehenden Ionen zur Wanderung bringt. An den Polen findet die Abscheidung der Elektrolyten nach Maßgabe der durchgegangenen Strommenge statt.

Man könnte nun für das Zustandekommen der galvanotropischen Krümmung die Ionenwanderung in der Lösung verantwortlich machen. Diese Größe ist schon verschiedentlich bestimmt worden. Sie beträgt z. B. für eine $8 \% \mathrm{CuSO}_{4}$-Lösung mit 1 Volt pro $\mathrm{m}-1,6 \cdot 10^{-8} \mathrm{~m}$ pro Sekunde $=0,00016 \mathrm{~mm}$. Oder pro Stunde $0,576 \mathrm{~mm}$ und für 12 Stdn. $6,912 \mathrm{~mm}$.

Bei den sehr schwachen zur Verwendung gekommenen Strömen ist diese Größe für die Beobachtungszeit von 12 Stunden so gering, daß man sie füglich vernachlässigen darf, ohne einen Fehler zu begehen. Meine Versuche zeigen dann deutlich, daß die Umstimmung bei den verschiedenen Stoffen nicht von der Ionenzahl, sondern von der stofflichen Zusammensetzung der Ionen abhängt. Jede Theorie, die sich auf die Verhältnisse der Ionenwanderung in der Lösung allein stützt, muß darum verlassen werden.

Von andern Möglichkeiten zur Erklärung des Galvanotropismus sind zwei wie mir scheint zu berücksichtigen.

Einmal ist es sicher, daß die Ionenwanderung nicht nur in der Lösung eintritt, sondern sie wird sich auch in jeder Zelle im Zellsaft zeigen, wenn die Wurzel in einen Stromkreis eingeführt wird, wie es in meinen Versuchen geschehen ist. Die Zellen der Wurzeln enthalten 
in ihrem Zellsaft immer eine ganze Reihe von Salzen gelöst, die gewiß zum Teil in die Ionen gespalten sind. Es werden bei der Ionenwanderung die einzelnen Ionen auf die Plasmahaut einen Druck ausüben, sofern diese den Austritt der Ionen verhindert. Soviel wir aber aus den osmotischen Verhältnissen wissen, ist das für die weitaus meisten Ionen der Fall.

Stellt man sich eine Zelle vor, deren Längsachse senkrecht zur Stromrichtung orientiert ist (Fig. 2), so wird dieser Druck, der durch die Ionenwanderung herbeigeführt wird, auf den beiden Flanken, die den Polen zugewendet sind, gleich sein, denn die Zahl der + und Ionen wird gleich sein. Einen einseitig stärkeren Druck auf einer Flanke, wie es etwa die Statholitentheorie für den Geotropismus annimmt, tritt nicht ein. Dieses Gleichgewicht würde nur dạnn verschoben, wenn durch Eintritt oder Austritt von Ionen auf den verschiedenen Flanken ungleiche Ionenmengen wirken würden. In diesem Falle würde die

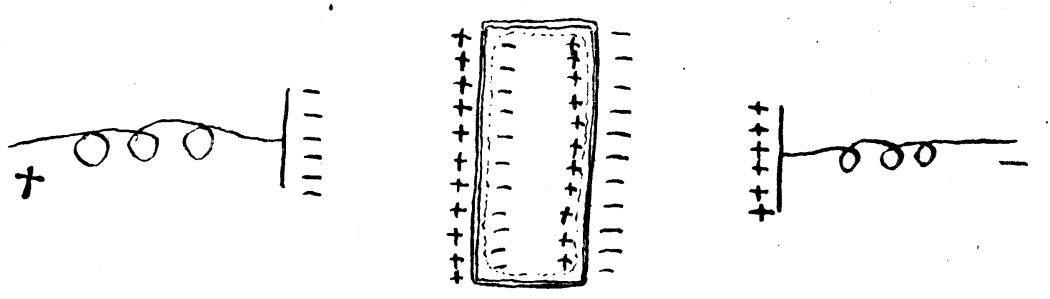

Fig. 2.

ganze Argumentation, die der Statolithentheorie des Geotropismus zugrunde liegt, für die Empfindung des elektrischen Reizes passen, nur mit dem Unterschiede, daß der Druck in unserem Falle durch ungleiche Verteilung der Ionen anstatt durch die Stärkekörner zustande kommt.

A. Coehn und W. Barrat haben vọn solchen Überlegungen ausgehend für die galvanotropischen Bewegungen von Paramaecium eine Erklärung zu geben versucht. Die Konzentration der Umstimmung der Lösung wäre somit von gleicher Ionenzahl, wie die Konzentration des Zellsaftes der empfindlichen Zelle. Nimmt man nun an, daß die Durchtrittsgeschwindigkeit von Kationen und Anionen durch den Plasmaschlauch eine ungleiche ist, so bekommt man eine ungleiche Ionenzahl und eine ungleiche elektrische Ladung auf den entsprechenden Flanken. Das Verhältnis wird sich ändern, wenn die Zahl der Ionen außen größer als innen ist in der einen Richtung und umgekehrt, wenn das Verhältnis innen größer als außen ist nach der anderen Richtung. Man kann sich die Umstimmung darnach erklären, doch befriedigt sie mich nicht. Es spricht dieser Auffassung vor allem die Tatsache entgegen, 
daß die Umstimmung bei den verschiedenen Salzen nicht bei gleichen Konzentrationen der Ionen liegt, was in diesem Falle eintreten müßte, wenn die Theorie richtig wäre. Dann müssen wir aber annehmen, daß die Plasmahaut durchaus gegen Elektrolyten kein indifferentes Gebilde ist, sondern durch diese verändert wird.

Welche Grundlagen für eine allfällige Erklärung des Galvanotropismus ergeben sich aus den Versuchen mit der Erbse?

Wir müssen hier vor allem festhalten, daß die Umstimmung der Wurzeln nicht durch die Stromstärke, sondern durch die Konzentration der Lösung bedingt wird, sofern wenigstens so schwache Ströme zur Verwendung gelangen, daß die Pflanze nicht geschädigt wird. Weiter zeigen die Versuche, daß die Umstimmung nicht proportional der Zahl der Moleküle oder Ionen bei den verschiedenen Salzen erfolgt. Die Reihenfolgen entsprechen aber ebenfalls nicht den Wanderungsgeschwindigkeiten der Ionen in wässerigen Lösungen.

Für eine Erklärung bleibt nur übrig anzunehmen, daß die Stoffe im .Protoplasma Veränderungen hervorrufen. Nur dann bekommen wir eine Erklärung für das gesetzmäßige Verhalten der verschiedenen Stoffe. Dieses beweist eine additive Eigenschaft. Man kann daraus folgern, daß Wirkung eines Salzes gleich ist der Wirkung der Kationen plus Wirkung der Anionen.

Wenn nun die Ionenwanderung die Ursache der Veränderungen ist, dann müssen wir annehmen, daß dadurch ein Eintritt von Ionen in die Zelle erfolgen und auf dem Wege des Durchtrittes durch die Plasmahaut eine Störung durch Ausflockungen oder Auflockerungen in diesem System kolloidaler Körper des Protoplasmas eintritt. Sofern Unterschiede in den beiden entgegengesetzten Flanken eintreten, wird eine Ungleichheit geschaffen, die zur Erzeugung eines Reizes völlig ausreichen würde.

Diese Ungleichheit kann ja eintreten durch die ungleiche Wanderungsgeschwindigkeit der Ionen beim Eintritt in die Plasmahaut; sie kann aber auch eintreten durch die ungleiche Ausfällung und Auflockerung der einzelnen Ionen im Plasma. Die Reihenfolgen der Wirkungen der Kationen und Anionen weisen mit aller Deutlichkeit darauf hin, daß solche Erscheinungen vertreten sind.

Nun wird aber der Eintritt der Kationen und Anionen auf beiden Flanken ungleich erfolgen, denn infolge der Wanderung der Ionen in der Außenlösung werden auf der einen Seite z. B. die Kationen der Wurzeln zu wandern, dann auf der entgegengesetzten Flanke von der Wurzel weg wandern und die Anionen genau in umgekehrter Richtung, 
Fig. 2. Dadurch wird jedenfalls eine weitere Ungleichheit geschaffen, die in der Wirkung einem ungleichen Eintritt der Ionen auf den entgegengesetzten Flanken der Wurzel gleich kommt.

Diese Verhältnisse werden auf das Zustandekommen der Krümmung gegenseitig sich beeinflussen und die Resultante dieser verschiedenen Wirkungen wird als Gesamtes den Reiz für die Wurzelkrümmung geben.

Charakteristisch für diese galvanotrope Wurzelkrümmung ist nun die Umstimmung der Wurzel, die wenigstens bei den schwachen Strömen unabhängig von der Stromintensität zu sein scheint. Diese Umstimmung führt uns zur Betrachtung einer andern Seite des Problems. Wie bereits erwähnt, erklären Coehn und W. Barrat diese Erscheinung bei den Paramäcien einfach dadurch, daß sie annehmen, daß im einen Falle die Ionenkonzentration außen größer als in der Zelle, im andern Falle umgekehrt ist. Die Gründe, die gegen eine solche Auffassung sprechen, habe ich bereits angeführt. Immerhin müssen wir aus allen bekannten Verhältnissen des Stoffaustausches schließen, daß, wenn ein Eintritt von Salzteilchen in die Zelle erfolgt, umgekehrt auch ein Austritt von Ionen aus dem Zellsaft in die Außenflüssigkeit eintritt. Diese durch die Plasmamembran durchtretenden Ionen werden in derselben die gleichen Veränderungen hervorrufen, wie die eintretenden Ionen und der labile Gleichgewichtszustand, in welchem die Plasmahaut sich befindet, kann nur das Resultat beider Wirkungen sein. Wenn man nach den Auffassungen des Stoffumsatzes annimmt, daß bei Eintritt von Ionen die gleiche Anzahl Ionen mit gleicher elektrischer Ladung austritt, wo der Gleichgewichtszustand hergestellt ist, so wird in der Plasmahaut eine Differenz sich nur ergeben aus den ungleichen Wirkungen elektrisch gleichgeladener Ionen auf das Plasma, indem auf den entgegengésetzten Seiten der Ionen Ein- und Austritt in verschiedener Weise erfolgen muß. Um die Umstimmung $\mathrm{zu}$ erklären, braucht man nur anzunehmen, daß die Unterschiedsschwelle infolge der Steigerung der Konzentration verschoben wird. Das eine Mal wird auf der einen Seite die Ausflockung kräftiger werden, das andere Mal auf der entgegengesetzten Seite. Dadurch wird die Reizung erklärt und die Umstimmung.

Ich sehe deshalb im Zustand der Umstimmung einen Gleichgewichtszustand, der auf beiden Flanken gleich ist. Sobald auf der einen Seite gegenüber der andern dieser Gleichgewichtszustand sich verschiebt, wird die Reizung eintreten. Es ist also die Empfindung einer Differenz, die sich zeigt und womit auch alle Erfahrungen mit der chemotropen Reizung übereinstimmen. 
Mit diesen Auseinandersetzungen befinde ich mich auf einem ähnlichen Boden wie J. Loeb in seiner Theorie des Galvanotropismus. Auch er nimmt die Wirkung der Ionen sowohl in der Außenlösung, als im Innern der Zellen an (innere und äußere Elektrolyse) und die Wirkung ist verschieden je nachdem die eine Wirkung oder die andere Form der Elektrolyse vertreten ist. Bei der Erbsenwurzel kommen, wie es mir sćheint, beide Wirkungen miteinander zur Geltung, die mit dem Durchtritt der Ionen durch die Plasmahaut verbunden sind. Weder das Șchema des Muskels (innere Elektrolyse), noch dasjenige der Hautdrüsenzéllen von Amblystoma und der Protozoen (äußere Elektrolyse) paßt für die Erbsenwurzel. Die Umstimmung findet bei allen Salzen mit Änderung der Konzentration statt, sie hat nach meiner Auffassung eine größere Bedeutung, als Loeb pag. 532 und 533 annimmt.

Nur durch die Wechselwirkung der Verhältnisse der inneren und äußeren Elektrolyse gelingt es, in befriedigender Weise für die Verhältnisse, wie sie bei der Erbsenwurzel vorliegen, eine Erklärung zu geben. Daß Stoffe ein- und austreten in diesem Falle, dürfte nach allem, was wir über den Stoffaustausch der Wurzeln kennen, keinem Zweifel unterliegen. Wenn man will ist deshalb die Anschauung, die ich für die Verhältnisse der Erbsenwurzel habe, nur eine besondere Anwendung der Loebschen Theorie des Galvanotropismus.

Wenn wir uns fragen, ob die beobachteten chemotropischen Reize, die verschiedene Salze auf die Wurzeln der Phanerogamen und auch auf die der Erbse ausüben, identisch sind mit den Reizerscheinungen des elektrischen Stromes, so können nur die Effekte der schwachen Ströme in Betracht kommen. Von diesen habe ich nachgewiesen, daß sie je nach der Konzentration der Lösung Krümmungen gegen die Kathode oder Anode auslösen. Chemotrope Erscheinungen treten nur in einem Konzentrationsgefälle auf und zwar wachsen die Wurzeln bei schwachen Konzentrationsdifferenzen gegen die stärkere Konzentration der Lösung, wie die Versuche von Newcombe und Rhodes, Lilienthal und Sammet zeigen.

In jedem Konzentrationsgefälle von Salzen treten elektrische Ströme infolge der ungleichen Wanderungsgeschwindigkeit der Ionen auf, die Konzentrationsströme. Diese verlaufen so, daß der Strom, der von der niedrigen Konzentration zur höheren geht, die positive Ladung bekommt, und der Strom, der von der höheren Konzentration zur niedrigen geht, die negative Ladung erhält. Wenn deshalb die Wurzelspitze in einem schwachen Konzentrationsgefälle sich zum Orte stärkerer Konzen- 
tration wendet, so ist das gleich bedeutend mit der Wendung zur Anode. Dieses tritt nur aber ebenfalls in der andern Anordnung des Experimentes im Galvanotropismus ein. Auch die in den Konzentrationsgefällen sich bildenden Ströme sind durchaus analog den ganz schwachen, an meinen Versuchen zur Anwendung gelangten Strömen. Nernst (pag. 708) berechnet die elektromotorische Kraft für das Konzentrationsgefälle $\mathrm{KCl} 0,1-0,01 \mathrm{Mol} \mathrm{zu} \mathrm{0,0542}$ Volt und die beobachtete Größe war 0,0532 Volt. Je nach dem Widerstand wird die zwischen zwei Punkten fließende Strommenge verschieden sein. Man kann aber leicht sehen, daß, wenn diese beiden Punkte, die eine Differenz von $0,1-0,01 \mathrm{Mol}$ aufweisen, in einem Konzentrationsgefälle nahe aneinanderrücken, der Widerstand immer kleiner und die durchfließende Strommenge größer wird. Größen von 0,00001-0,000001 Amp. treten bei Entfernung dieser Punkte leicht auf. Daraus kann man wohl mit Recht schließen, daß bei den Versuchen über chemotropische Reizung der durch das Konzentrationsgefälle erzeugte Strom, wie ihn unter den Bedingungen die Lilienfeld, Shibata, Sammet eingehalten haben, in der Größe analog demjenigen ist, den ich zu meinen Versuchen wählte.

Die Betrachtungen über die Konzentrationsströme und die chemotrope Reizung der Wurzeln in Konzentrationsgefällen führen mich aber notwendig zur Frage über die Ursache des Reizes, der die Wurzel zur Ablenkung der Wachstumsrichtung verursacht. In einer homogenen Salzlösung tritt eine Ablenkung der Wurzeln in ihrer Wachstumsrichtung nicht ein, sie folgt dem Geotropismus. Sobald ein Strom durchgeht, beginnt die Ablenkung. Es ist somit nur die Wanderung der Ionen eingetreten; nur diese kann die Ablenkung verursacht haben. Zum gleichen Resultate gelangt man aber für die Verhältnisse bei chemotroper Reizung im Konzentrationsgefälle. Gegenüber der homogenen Lösung zeigt das Konzentrationsgefälle nur die Wanderung der Ionen und das damit verbundene Auftreten der Konzentrationsströme.

Daraus schließe ich, daß Chemotropismus der Salze und Galvanotropismus bei den Wurzeln identische Erscheinungen sind. Beide werden hervorgerufen durch die Ionenwanderung - und die damit verbundenen Veränderungen in der Phanerogamenwurzel; in dem einen Falle wird aber die Ionenwanderung durch ein Konzentrationsgefälle, im andern Falle durch den elektrischen Strom herbeigeführt.

Wenn diese Sätze richtig sind, dann ist aber zugleich die Prüfung der Wurzeln in einem Stromkreis unter den bis jetzt bekannten das beste Mittel, um die Konzentration der Umstimmung genau festzu- 
stellen. Bei Phanerogamenwurzeln ist außer meinen Versuchen nur noch von Sammet der Versuch gemacht worden, diese Konzentration $\mathrm{zu}$ ermitteln. Indes geben seine Versuche leider keine exakten Anhaltspunkte, um diese Differenz zu bestimmen. Soviel ich aus seinen Versuchsresultaten ersehe. muß das Ergebnis annähernd für $\mathrm{NaCl}, \mathrm{KNO}_{3}$ dasselbe sein. wie ich es erhalten habe. Die Versuche mit Gelatineblöcken, wie sie Lilienfeld angewendet hat, sind leider gar nicht geeignet, für diese Fragen verwendet zu werden, indem die Kolloide wie Gelatine und andere sehr stark hemmend auf die Ionenwanderung und damit auf die Ströme wirken. Auch in den Resultaten mit seinen Versuchen finden sich je nach den Umständen der Versuchsanstellung ungleiche Resultate. Das beste Beispiel, um zu zeigen, wie verschieden die Resultate ausfallen je nach der verwendeten Methode, sind die Zuckerarten. Nach Sammet geben Glykose und Rohrzucker eine chemotrope Reizung, nach Lilienfeld geben sie keine Reizung auf Wurzeln!

Mit Nichtelektrolyten habe ich nur einige wenige Versuche gemacht. Ein Zusatz von Rohrzucker, Glyzerin zu einer schwachen Salzlösung zeigt keine Einwirkung der Nichtelektrolyten. Die Wurzeln krümmen sich nur, wie wenn das Salz allein wirken würde. Indes gebe ich gerne $\mathrm{zu}$, daß meine Versuchsanstellung nicht geeignet ist, diese Frage zu lösen.

Wenn Nichtelektrolyte einen Chemotropismus auslösen - und daran ist, wie mir scheint, kein Zweifel möglich, - so ist die Wirkung des Nichtelektrolyten in den Veränderungen der Permeabilitätsverhältnisse der Plasmamembran zu suchen. Wird durch eine Einwirkung von ungleichen Mengen Nichtelektrolyten auf den entgegengesetzten Seiten der Wurzel die Plasmahaut nur etwas geändert in ihren Permeabilitätsverhältnissen gegenüber den im Innern der Zelle enthaltenen Salzen, die - sicher zum Teil dissoziert sind, so werden wir auf den entgegengesetzten Seiten ungleiche ausfällende und auflockernde Wirkungen auf die Kolloide des Plasmas haben und dementsprechend die Reizung. Daraus ist aber auch die Umstimmung leicht $\mathrm{zu}$ verstehen und $\mathrm{zu}$ erklären. Es kann nicht in meiner Aufgabe liegen, hier diese Verhältnisse der Nichtelektrolyte näher zu erörtern, bemerken will ich nur, daß sie mit meiner Auffassung des Zustandekommens der Reizung bei den Elektrolyten in Einklang stehen.

Es bleibt mir nur übrig, einen Blick auf die nachgewiesene, besonders ausgebildete Empfindlichkeit der Wurzelspitze zu werfen. Die Untersuchungen über den statischen Apparat der Wurzelspitze haben ergeben, daß die besonders differenzierten Sinneszellen mit einem einzigen 
großen Zellenraum ausgestattet sind, der mit dünnflüssigem Zellsaft erfüllt ist. In einer solchen Zelle muß die Wanderung der Ionen im Zellsaft leichter vor sich gehen, als wenn der Raum in viele kleine Vacuolen abgeteilt ist. Die Wirkung der Ionenwanderung wird deshalb in der Region der Wurzelspitze besonders gut sich zeigen. Damit steht im Einklang die besondere Empfindlichkeit der Wurzelspitze. Die rückwärts liegenden Partien der Wurzel sind zwar ebenfalls befähigt, den Reiz aufzunehmen, wenn auch in viel schwächerem Maße. Das zeigt aber nur, daß in allen Zellen Reiz empfunden wird, infolge besonders günstiger Verhältnisse in der Wurzelspitze jedoch stärker als in den rückwärts liegenden Partien der Wurzel.

Mit anästhesierenden Mitteln habe ich nur wenig Versuche gemacht. Es zeigt sich, daß es gelingt, die Empfindlichkeit für den galvanotropischen Reiz aufzuheben. Bei einem Zusatz von 20\% Chloroformwasser zeigten die Wurzeln noch Wachstum, hingegen trat die Ablenkung der Wachstumsrichtung der Wurzel nicht mehr ein bei $0,1 \% \mathrm{KCl}$ und $0,05 \% \mathrm{KCl}$. Der Versuch zeigt uns, daß es gelingt, die Reizempfindlichkeit der Wurzel aufzuheben, ohne daß ihr Wachstum sistiert wird.

Meine Untersuchungen beziehen sich auf die Erbsenwurzel. Ich hielt es für richtiger, einen Lebensvorgang an einem Objekte möglichst intensiv zu studieren, als gleichzeitig mit zahlreichen Pflanzen zu operieren. Ich war um so mehr gezwungen, diesen Weg einzuhalten, als Raum und Hilfsmittel, die mir zur Verfügung standen, recht beschränkte waren. Dennoch habe ich nicht versäumt, mich zu überzeugen, daß die Ablenkung der Wurzeln durch schwache elektrische Ströme eine ganz allgemein verbreitete Erscheinung ist. Ich habe sie bei folgenden Pflanzen beobachtet: Lemna trisulca, Allium cepa, Hyacinthus orientalis, Zea Mays, Triticum vulgare, Secale cereale, Avena sativa, Hordeum vulgare, Lepidium sativum, Bassica rapa und oleracea, Pisum sativum, Phaseolus vulgaris, Lupinus hirsutus, albus, Ricinus communis, Cucurbita Pepo, Lactuca scariola, Cichorium Intybus.

Sie kommt auch bei den Kryptogamen vor. So konnte ich sie sehen bei Bacillus fluorescens, bei Pandorina morum. Ich zweifle nicht, daß die Spermatozoiden der Moose und Farne auch auf diese Weise gelenkt werden können.

Die Zahlen für die Konzentrationen der Umstimmung werden für die einzelnen Objekte gewiß verschieden ausfallen, und es wird Aufgabe weiterer Untersuchungen, diese besonders noch festzustellen. 
Der spezifische Reizstoff wird bei den betreffenden Objekten jeweils eine besonders niedrige Konzentration der Wirkung und Umstimmung aufweisen.

\section{Zusammenfassung der Resultate.}

Bei den Wurzeln sind die Wirkungen starker und schwacher elektrischer Ströme zu unterscheiden. Die einen rufen Abtötungserscheinungen oder Wachstumsstörungen hervor und bewirken auf diese Weise eine Ablenkung der Wachstumsrichtung der Wurzeln. Die andern erzeugen Ablenkungen der Wachstumsrichtung der Wurzeln ohne wesentliche Störung des Wachstums.

Die Umstimmung der Wachstumsrichtung der Wurzeln ist abhängig von der Konzentration der verwendeten Lösung, wenn man nur die Wirkungen der schwachen Ströme betrachtet. Diese Umstimmung tritt bei allen geprüften Salzen ein; die Konzentration der Umstimmung aber ist von Salz zu Salz verschieden. Es ergibt sich immerhin eine Regelmäßigkeit, so daß die Wirkung der Salze auf die Wachstumsrichtung als eine additive Eigenschaft aufgefaßt werden muß, die gleich ist der Summe der Wirkung der Kationen plus Wirkung der Anionen.

Galvanotropismus und Chemotropismus der Salze sind auf gleiche Ursachen (elektrische Ströme und die damit verbundene Ionenwanderung) zurückzuführen.

- Beide Erscheinungen treten in ganz allgemeiner Verbreitung bei den Phanerogamenwurzeln auf.

Es sei mir gestattet, Herrn Prof. Dr. P. Weiß, sowie seinem Assistenten Herrn Dr. J. Kunz für die zahlreichen Ratschläge, sowie für die Benutzung der Apparate des physikalischen Institutes des eidgenössischen Polytechnikums an dieser Stelle bestens zu danken.

\section{Literaturverzeichnis.}

1. J. Brunchorst, Über die Funktion der Spitze bei den Richtungsbewegungen der Wurzeln. II. Galvanotropismus. Ber. der deutschen bot. Gesellsch. 1884 ; Bd. II.

2. Ders., Zur Frage über den sogenannten Galvanotropismus. Bot. Zentralblatt 1885, Bd. XXIII.

3. Ders., Notizen über den Galvanotropismus. Bergens Museums Aarsberetining 1889.

4. R. Buller, Contributions to our knowledge of the physiology of the spermatozoa of ferns. Annals of Botany 1900, Bd. XIV. 
5. Alfred Coehn und Wakelin Baratt, Über Galvanotaxis vom Standpunkt der physikalischen Chemie. Zeitschr. f. allg. Physiologie 1905, H. 1.

6. Elfving, Über eine Wirkung des galvanischen Stromes auf wachsende Wurzeln. Bot. Zeitung 1882.

7. Lilienfeld, Über den Chemotropismus der Wurzeln, Beih. z. bot. Zentralbl. 1905, Bd. XIX.

8. Ders., Über den Chemotropismus der Wurzeln. Vorläufige Mitteilung. Ber. d. deutschen bot. Gesellsch. 1905.

9. B. Lidfors, Über den Chemotropismus der Marchantiaspermatozoiden. Jahrb. f. wissensch. Bot. 1905 .

10. J. Loeb, Zur Theorie des Galvanotropismus. Pflügers Archiv 1897, Bd. LXV; IV. Über die Ausscheidung elektropositiver Ionen an der äußeren Anodenfläche protoplasmatischer Gebilde als Ursache der Abweichungen vom Pflügerschen Erregungsgesetz von J. Loeb und S. P. Budgett.

11. J. Müller-Hettlingen, Über galvanische Erscheinungen an keímenden Samen. Pflügers Archiv f. Physiologie 1883, Bd. XXXI.

12. Nernst, Theoretische Chemie 1905, III. Aufl.

13. Newcombe und Rhodes, Chemotropism of roots. Botanical Gazette 1904, Vol. XXXVII.

14. W. Pfeffer, Lokomotorische Richtungsbewegungen durch chemische Reize. Unters. a. d. bot. Institut Tübingen 1884, Bd. I.

15. Ders., Über chemotaktische Bewegungen von Bakterien, Flagellaten und Volvocineen, Unters. a. d. bot. Institut Tübingen 1888, Bd. II.

16. W. Rothert, Beobachtungen und Betrachtungen über taktische Reizerscheinungen. Flora 1901, Bd. LXXXVII.

17. R. Sammet, Untersuchungen über Chemotropismus und verwandte Erscheinungen bei Wurzeln, Sprossen und Pilzfäden. Jahrb. f. wissensch. Botanik 1905.

18. K. Shibata, Studien über die Chemotaxis der Isoëtesspermatozoiden. Jahrb. f. Botanik 1905. 